International Journal of Modern Physics A,

(C) World Scientific Publishing Company

\title{
TYPE IIB ORIENTIFOLDS WITH DISCRETE TORSION
}

\author{
ROBERT L. KARP ${ }^{*}$ F. PAUL ESPOSITO, LOUIS WITTEN \\ Physics Department, University of Cincinnati \\ Cincinnati, Ohio 45221-0011, USA
}

\begin{abstract}
We consider compact four-dimensional $\mathbf{Z}_{\mathbf{N}} \times \mathbf{Z}_{\mathbf{M}}$ type IIB orientifolds, for certain values of $N$ and $M$. We allow the additional feature of discrete torsion and discuss the modification of the consistency conditions arising from tadpole cancellation. We point out the differences between the cases with and without discrete torsion.
\end{abstract}

Orientifold compactifications $\$$ of the type IIB superstring circumvent the problem that the type I theory does not produce a chiral spectrum when compactified on a Calabi-Yau threefold with standard embedding of the gauge degrees of freedom. Independently of this discrete torsion (DT) was introduced as a phase factor related to the B-field, allowed by modular invariance 2 in orbifold compactifications of the closed string theories. In the open string theories the analogous notion of DT was discovered relatively recently, only after D-branes were better understood 3 . In addition the relationship between closed and open DT has been further clarified 1 .

The pioneering work for $\mathbf{Z}_{\mathbf{2}}$ orientifolds was quickly generalized to $\mathbf{Z}_{\mathbf{n}}$ for different values of $n$ 's question of noncompact orientifolds with DT was addressed as well aspects of DT was partly described 9 and there has recently been a revival of interest in the subject 10 .

The complete orientifold group we consider here is $G_{1}+\Omega G_{2}$ with $\Omega h \Omega h^{\prime} \in G_{1}$ for $h, h^{\prime} \in G_{2}$. We restrict our attention to $G_{1}=G_{2}=\mathbf{Z}_{\mathbf{N}} \times \mathbf{Z}_{\mathbf{M}}$. The generator of either of the factors will have the form $\theta=\exp \left(2 i \pi\left(v_{1} J_{45}+v_{2} J_{67}+v_{3} J_{89}\right)\right)$, with $J_{m n}$ the $S O(6)$ Cartan generators, acting on the compact $T^{6}$ (complexified) coordinates $Z_{1}=X_{4}+i X_{5}, Z_{2}=X_{6}+i X_{7}$ and $Z_{3}=X_{8}+i X_{9}$ as $\theta Z_{i}=\mathrm{e}^{2 i \pi v_{i}} Z_{i}$. If we chose the twist vectors of the $\mathbf{Z}_{\mathbf{N}}$ and $\mathbf{Z}_{\mathbf{M}}$ generators $\theta$ and $\omega$ to be of the form $v_{\theta}=v=\frac{1}{N}(1,-1,0)$ and $v_{\omega}=w=\frac{1}{M}(0,1,-1)$, we end up with $\mathrm{N}=1 \mathrm{~d}=4$ supersymmetry. Undoubtedly, there are many equally interesting choices that do not have this form.

To derive the massless spectra we work in light-cone gauge. The GSO projected untwisted massless Ramond states $\left|s_{0} s_{1} s_{2} s_{3}\right\rangle$ transform as $\theta\left|s_{0} s_{1} s_{2} s_{3}\right\rangle=$ $\mathrm{e}^{2 i \pi v \cdot s}\left|s_{0} s_{1} s_{2} s_{3}\right\rangle$.

In this paper we will be mainly interested in the Klein bottle vacuum to vacuum amplitude; the Mobius strip and the cylinder in fact have similar expression. The

*Present address: Department of Mathematics, Duke University, Durham, NC 27708 
Klein bottle amplitude is given by

$$
\mathcal{K}=\frac{V_{4}}{2 M N} \sum_{g, h} \int_{0}^{\infty} \frac{d t}{2 t}\left(4 \pi^{2} \alpha^{\prime} t\right)^{-2} \operatorname{Tr}_{h}\left\{\frac{1+(-1)^{F}}{2} \Omega g \mathrm{e}^{-2 \pi t\left[L_{0}(h)+\tilde{L}_{0}(h)\right]}\right\},
$$

where the sums run over the entire group $G_{1}=\mathbf{Z}_{\mathbf{N}} \times \mathbf{Z}_{\mathbf{M}}$, and the trace is computed in the sector twisted by $h$. As any element of $G_{1}$ is of the form $x^{a} y^{b}$, where $x(y)$ is a generator of $\mathbf{Z}_{\mathbf{N}}\left(\mathbf{Z}_{\mathbf{M}}\right)$, and $\Omega$ interchanges the sectors twisted by $x^{a} y^{b}$ and $x^{N-a} y^{M-b}$, we see immediately that in order to have DT make a difference in the Klein bottle amplitude we must require that either $N$ or $M$ is even, and we have to study the sector twisted by $x^{a} y^{b}$, with $(a, b)$ taken from the set $\{(N / 2,0),(0, M / 2),(N / 2, M / 2)\}$. Due to space limitation we can only hint at the form of the expressions involved. For example for the Klein bottle amplitude in the sector twisted by $x^{N / 2}$ we have:

$$
\mathcal{K}\left(x^{\frac{N}{2}}, x^{a} y^{b}\right)=\chi_{\left(\frac{N}{2}, 0\right)}^{(a, b)} \sum_{\alpha, \beta=0}^{\frac{1}{2}} \eta_{\alpha, \beta} \frac{\vartheta\left[\begin{array}{c}
\alpha \\
\beta
\end{array}\right]}{\eta^{3}}\left(\prod_{i=1}^{2} \frac{\vartheta\left[\begin{array}{c}
\alpha+\frac{1}{2} \\
\beta+2 u_{i}
\end{array}\right]}{\vartheta\left[\begin{array}{c}
\frac{1}{2}+2 u_{i} \\
2
\end{array}\right]}\right)\left(-2 \sin 2 \pi u_{3}\right) \frac{\vartheta\left[\begin{array}{c}
\alpha \\
\beta+2 u_{3}
\end{array}\right]}{\vartheta\left[\begin{array}{c}
\frac{1}{2} \\
\frac{1}{2}+2 u_{3}
\end{array}\right]}
$$

where $u_{i}=a v_{i}+b w_{i}$, and the argument of the $\vartheta^{\prime} \mathrm{s}$ and $\eta$ 's is $e^{-4 \pi t}$. In addition to this we also have factors coming from the compact momenta and from windings 1 . Using the properties of $\vartheta$ functions Eq. (2) can be simplified dramatically:

$$
\mathcal{K}\left(x^{\frac{N}{2}}, x^{a} y^{b}\right)=(1-1) \chi_{\left(\frac{N}{2}, 0\right)}^{(a, b)} \frac{\vartheta\left[\begin{array}{c}
0 \\
\frac{1}{2}
\end{array}\right]}{\eta^{3}}\left(\prod_{i=1}^{2} \frac{\vartheta\left[\begin{array}{c}
\frac{1}{2} \\
\frac{1}{2}+2 u_{i}
\end{array}\right]}{0\left[\begin{array}{c}
0 \\
\frac{1}{2}+2 u_{i}
\end{array}\right]}\right)\left(-2 \sin 2 \pi u_{3}\right) \frac{\vartheta\left[\begin{array}{c}
0 \\
\frac{1}{2}+2 u_{3}
\end{array}\right]}{\vartheta\left[\begin{array}{c}
\frac{1}{2} \\
\frac{1}{2}+2 u_{3}
\end{array}\right]},
$$

In what follows we focus on the differences in the closed string sector arising from the presence of DT. For $\mathbf{Z}_{\mathbf{n}} \times \mathbf{Z}_{\mathbf{m}}$ with $n$ and $m$ odd, though DT is possible, it cannot contribute to the Klein bottle amplitude, as the relevant twisted sector amplitudes are zero. This implies that for these models there is no difference in the tadpole cancellation equation with or without DT. As the orientifolds constructed in these cases were based on projective representations on the Chan-Paton indices, we conclude that DT has no effect.

The next simplest class of models is $\mathbf{Z}_{\mathbf{2}} \times \mathbf{Z}_{\mathbf{m}}$. Here there are two sub-cases. For $m$ odd there is no DT. For $m$ even we can take $x$ (resp. $y$ ) as the generator of $\mathbf{Z}_{\mathbf{2}}$ (resp. $\mathbf{Z}_{\mathbf{m}}$ ), and $\omega_{2}=-1$ as the generator of $H^{2}\left(\mathbf{Z}_{\mathbf{2}} \times \mathbf{Z}_{\mathbf{m}}, U(1)\right)$. For the three potentially nonzero amplitudes we get: $\mathcal{K}(x,-)=\mathcal{K}\left(x y^{m / 2},-\right)=0$, and $\mathcal{K}\left(y^{m / 2}, x^{a} y^{b}\right) \neq 0$ iff $2 b / m \notin \mathbf{Z}$. Analyzing the different $m$ 's is easy again. For $m=2: 2 b / m=b$ and this is integer, so all the twisted sector has zero amplitude. For $m=4$ we have the $b=1,3$ nonzero amplitudes, but $\epsilon\left(y^{2}, x^{a} y^{b}\right)=1$, and DT has no effect. More generally for $m=4 l: \epsilon\left(y^{2 l}, x^{a} y^{b}\right)=1$ and we see that the $\mathbf{Z}_{\mathbf{2}} \times \mathbf{Z}_{\mathbf{4 l}}$ orbifold has the same tadpole cancellation condition with and without DT. The case $m=6$ requires more work to see what happens.

For the $\mathbf{Z}_{\mathbf{2}} \times \mathbf{Z}_{\mathbf{6}}$ orientifold the sectors we are interested in are the ones twisted by $x, x y^{3}, y^{3}$. It may easily be seen that the sector twisted by $y^{3}$ is the only 
nonzero one. In what follows we focus on the $\mathcal{K}\left(y^{3}, x y^{a}\right)=0$ contributions, which will be proportional to $1 / V_{1}$, as opposed to the $\mathcal{K}\left(y^{3}, y^{a}\right)$ contributions that are in fact proportional to $V_{1}$. We also have $\epsilon\left(y^{3}, x^{a} y^{b}\right)=(-1)^{a}$. It turns out that $\mathcal{K}\left(y^{3}, x y^{a}\right)=0$ for $a=0,3$, while for other values of $a$ they all equal a common value proportional to $\vartheta\left[\begin{array}{c}\frac{1}{2} \\ -\frac{1}{6}\end{array}\right] \vartheta\left[\begin{array}{c}\frac{1}{2} \\ \frac{1}{6}\end{array}\right] / \vartheta\left[\begin{array}{c}0 \\ -\frac{1}{6}\end{array}\right] \vartheta\left[\begin{array}{c}0 \\ \frac{1}{6}\end{array}\right]$. In the limit $t \rightarrow 0$ the twisted Klein bottle amplitudes will give the contribution $(2 t)\left(64 \pi^{2} \alpha^{\prime}\right) / V_{1}$ without DT, and the negative of this with DT. Similarly, the untwisted Klein bottle contribution that contributes with a factor of $1 / V_{1}$ turns out to be $\mathcal{K}\left(1, x y^{a}\right)$, for $a=1,3,4,5$. In the $t \rightarrow 0$ limit these add up the contribution $3(2 t)\left(64 \pi^{2} \alpha^{\prime}\right) / V_{1}$. Thus in the case without DT we have a tadpole contribution proportional to $(2 t)\left(256 \pi^{2} \alpha^{\prime}\right) / V_{1}$, which turns gut to require $32 \mathrm{D}$-branes to be canceled. This agrees with the already known result 7 . On the other hand, for the case with DT the tadpole cannot be canceled, rendering the model perturbatively inconsistent, in the sense ofl1.

The next interesting case is $\mathbf{Z}_{\mathbf{3}} \times \mathbf{Z}_{\mathbf{6}}$. More generally for $n$ odd the $\mathbf{Z}_{\mathbf{n}} \times \mathbf{Z}_{\mathbf{2 n}}$ DT is $\epsilon\left(y^{n}, x^{a} y^{b}\right)=e^{(2 \pi i / n) n(-b)}=1$, and once again DT has no effect.

The $\mathbf{Z}_{\mathbf{4}} \times \mathbf{Z}_{\mathbf{4}}$ model is interesting to analyze as well. It is was known that without DT this model was perturbatively inconsistent. Our hope was that DT would change the tadpole cancellation conditions, and allow for a consistent solution. It is elementary to show that $\epsilon\left(x^{2}, x^{a} y^{b}\right) \neq 1$ iff $b=1,3 ; \epsilon\left(x^{2} y^{2}, x^{a} y^{b}\right) \neq 1$ iff $a-b=-3,-1,1,3$, and $\epsilon\left(y^{2}, x^{a} y^{b}\right) \neq 1$ iff $a=1,3$. Unfortunately it turns out that with these constraints $\mathcal{K}\left(x^{2},-\right)=\mathcal{K}\left(x^{2} y^{2},-\right)=0$, and $\mathcal{K}\left(y^{2},-\right)=0$, implying that even by turning on DT we cannot perturbatively save the model.

\section{Acknowledgments}

R.L.K. would like to thank R.G. Leigh for useful conversations. This work was supported in part by the Department of Energy under the contract number DOEFGO2-84ER40153. RLK was also supported in part by the National Science Foundation grant DMS-9983320.

\section{Note}

After this talk was given an exhaustive treatment of the subject appeared 12 that overlaps partly with our results. 
Type IIB orientifolds with discrete torsion

\section{References}

1. A. Sagnotti, Talk presented at the Cargese Summer Institute on Non-Perturbative Methods in Field Theory, 1987. P. Horava, Nucl. Phys. B 327, 461 (1989). Phys. Lett. B 231, 251 (1989). E. G. Gimon and J. Polchinski, Phys. Rev. D54, 1667 (1996) hep-th/9601038. G. Pradisi and A. Sagnotti, Phys. Lett. B 216, 59 (1989).

2. C. Vafa, Nucl. Phys. B273, 592 (1986). NUPHA,B273,592;

3. M. R. Douglas, hep-th/9807235, hep-th/9903031.

4. J. Gomis, JHEP 0005, 006 (2000) hep-th/0001200].

5. M. Bianchi and A. Sagnotti, Phys. Lett. B 247, 517 (1990). Nucl. Phys. B 361, 519 (1991). E. G. Gimon and C. V. Johnson, Nucl. Phys. B477, 715 (1996) hepth/9604129. G. Aldazabal et al. Nucl. Phys. B536, 29 (1998) hep-th/9804026.

6. M. Berkooz and R. G. Leigh, Nucl. Phys. B483, 187 (1997) hep-th/9605049].

7. G. Zwart, Nucl. Phys. B526, 378 (1998) hep-th/9708040.

8. M. Klein and R. Rabadan, JHEP 0007, 040 (2000) thep-th/0002103.

9. E. R. Sharpe, hep-th/9909108, hep-th/9909120.

10. E. R. Sharpe, hep-th/0008154, 0008170, 0008184. M. R. Gaberdiel, hep-th/0008230. P. S. Aspinwall and M. R. Plesser, hep-th/0009042. P. S. Aspinwall, hep-th/0009045. P. Bantay, hep-th/0004025.

11. Z. Kakushadze et al, Nucl. Phys. B533, 25 (1998) hep-th/9804092.

12. M. Klein and R. Rabadan, hep-th/0008173. 